\title{
Correction: A novel NDUFS3 mutation in a Chinese patient with severe Leigh syndrome
}

\author{
Xiaoting Lou ${ }^{1} \cdot$ Hao Shi ${ }^{1} \cdot$ Shumeng Wen ${ }^{1} \cdot$ Yuanyuan $\mathrm{Li}^{1} \cdot \mathrm{Xiujuan} \mathrm{Wei}^{1} \cdot \mathrm{Jie} \mathrm{Xie}^{1} \cdot \mathrm{Lin} \mathrm{Ma}^{2} \cdot \mathrm{Yanling} \mathrm{Yang}^{3}$. \\ Hezhi Fang ${ }^{1} \cdot$ Jianxin Lyu $\mathbb{1}^{4}$
}

Published online: 28 September 2018

(c) The Author(s) under exclusive licence to The Japan Society of Human Genetics 2018

Correction to: Journal of Human Genetics https://doi.org/ 10.1038/s10038-018-0505-0; published online 23 August 2018

The originally published version of this article contained an error in Fig. 1. The correct figure of this article should have read as below. This has now been corrected in the PDF and HTML versions of the article. The authors apologize for any inconvenience caused.

Yanling Yang

organic.acid@126.com

$\triangle$ Hezhi Fang

FangH@wmu.edu.cn

1 Key Laboratory of Laboratory Medicine, Ministry of Education, Zhejiang Provincial Key Laboratory of Medical Genetics, School of Laboratory Medicine and Life sciences, Wenzhou Medical University, 325035 Wenzhou, Zhejiang, China

2 Department of Clinical Laboratory, The First Affiliated Hospital of Zhengzhou University, Key Clinical Laboratory of Henan, ProvinceZhengzhou, Henan, China

3 Department of Pediatrics, Peking University First Hospital, 100034 Beijing, China

4 Zhejiang Provincial People's Hospital, Affiliated People's Hospital of Hangzhou Medical College, 310053

Hangzhou, Zhejiang, China 
A
c. $418 \mathrm{C}>\mathrm{T}$
p.R140W
c.595C $>\mathrm{T}$
p.R199W

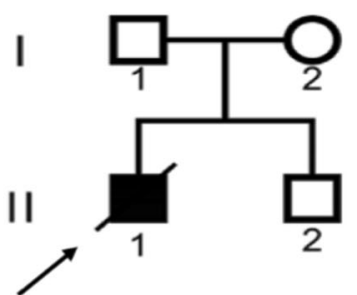

C

Mus musculus Rattus norvegicus Homo sapiens

Pan troglodytes Canis lupus familiaris Bos taurus

Gallus domesticus

Danio rerio

Serpula lacrymans Lctalurus punctatus Nothobranchius furzer

Salmo salar c. $595 \mathrm{C}>\mathrm{T}$

B

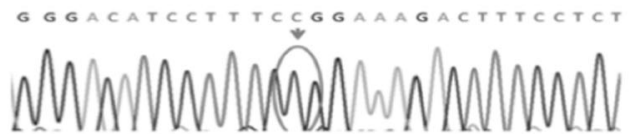

G GGACATCCTTTCCGGAAAGACTTTCCTCT

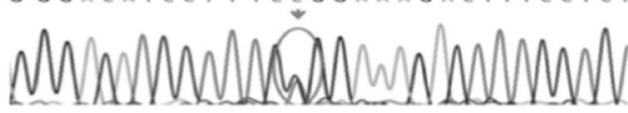

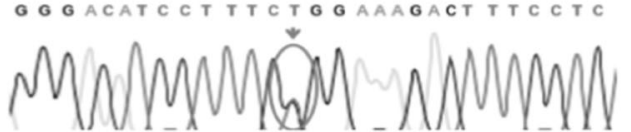

\begin{tabular}{l}
\multicolumn{1}{c}{ p.R140W } \\
\hline SLRFNSRIRVKTY \\
SLRFNSRIRVKTY \\
SLRFNSRIRVKTY \\
SLRFNSIRVKTY \\
SLRFNRIRVKTY \\
SLRFNSRIRVKTY \\
SLRFNSRIRVKTY \\
SLHYNSRIRVKTY \\
SI KLAGRIRVKTY \\
SLRFNSRIRVKTY \\
SLRYSRIRIKTY \\
SLRYNSRIRLKTY \\
\hline
\end{tabular}

Mus musculus

Rattus norvegicus

Homo sapiens

Pan troglodytes

Canis Iupus familiaris

Bos taurus

Gallus domesticus

Danio rerio

Serpula lacrymans

Lctalurus punctatus

Nothobranchius furzer

Salmo salar

c. $418 \mathrm{C}>\mathrm{T}$

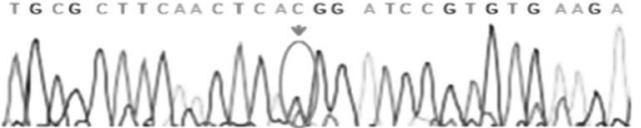

Control 2<smiles>[3H][Te]OC1CC1</smiles>
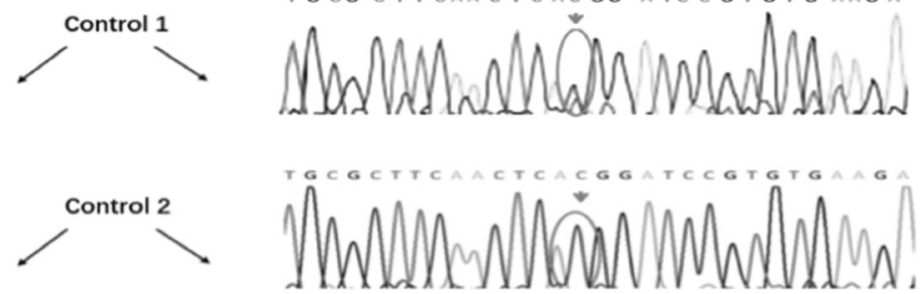

p.R199W

FEGHPFRKDFPL

FEGHPFRKDFPL

FEGHPFRKDFPL

FEGHPFRKDFPL

FEGHPFRKDFPL

FEGHPFRKDFPL

FEGHPFRKDFPL

FEGHPFRKDFPL

FEGHPLRKDFPL

FEGHPFRKDFPL

FEGHPFRKDFPL

FEGHPFRKDFPL

Patient

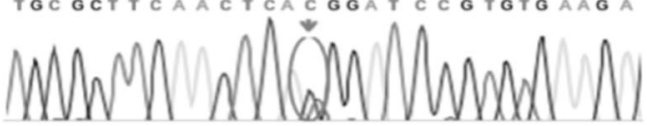

Fig. 1 